\title{
Exosome-mimetic nanoplatforms for targeted cancer drug delivery
}

\author{
Abi J. Vázquez-Ríos 1,2,5, Ángela Molina-Crespo 3,5, Belén L. Bouzo 1,2, Rafael López-López, \\ Gema Moreno-Bueno ${ }^{4,5}$ and María de la Fuente ${ }^{1,5^{*}}$ (1)
}

\begin{abstract}
Background: Lack of effective tumor-specific delivery systems remains an unmet clinical challenge for successful translation of innovative therapies, such as, therapeutic oligonucleotides. In the past decade, exosomes have been suggested to be ideal drug delivery systems with application in a broad range of pathologies including cancer, due to their organotropic properties. Tumor-derived exosomes, having tumor-homing properties, can efficiently reach cancer cells and therefore behave as carriers for improved drug delivery to the primary tumor and metastases. However, due to their complex composition, and still undefined biological functions, safety concerns arise hampering their translation to the clinics.

Results: We propose here the development of exosome-mimetic nanosystems (EMNs) that simulate natural tumor-derived exosomes with respect to their structure and functionality, but with a controlled composition, for the targeted delivery of therapeutic oligonucleotides to lung adenocarcinoma cells (microRNA-145 mimics). Making use of the well-known liposome technology, EMNs can be engineered, loaded with the therapeutic compounds, and tailored with specific proteins (integrin a6 $\beta 4$ ) providing them organotropic properties. EMNs show great similarities to natural exosomes with respect to their physicochemical properties, drug loading capacity, and ability to interact with the cancer target cells in vitro and in vivo, but are easier to manufacture, can be produced at high yields, and are safer by definition.
\end{abstract}

Conclusions: We have designed a multifunctional nanoplatform mimicking exosomes, EMNs, and proved their potential to reach cancer cells with a similar efficient that tumor-derived exosomes but providing important advantages in terms of production methodology and regulations. Additionally, EMNs are highly versatile systems that can be tunable for a broader range of applications.

Keywords: Exosomes, Exosome-mimetic nanoplatforms, Biomimetics, Drug delivery systems, Gene therapy, Cancer

\section{Background}

Recent data regarding the outcome of cancer highlight the need for more effective and innovative therapies. It is generally accepted that selectively reaching tumor cells will allow for more effective treatments without toxic side effects [1]. Given their role in intercellular communication, exosomes fulfill the requirements of an ideal drug delivery system. They can (i) transport molecules, (ii)

\footnotetext{
${ }^{*}$ Correspondence: maria.de.la.fuente.freire@sergas.es

${ }^{1}$ Nano-Oncology Unit, Translational Medical Oncology Group, Health Research Institute of Santiago de Compostela (IDIS), SERGAS, Choupana Street s/n, 15706 Santiago de Compostela, Spain

Full list of author information is available at the end of the article
}

cross biological membranes, (iii) overcome peripheral macrophages, and (iv) reach specific cell types to release their content [2]. Exosomes loaded with anticancer drugs have already shown promise as a new therapeutic approach in animal models [3-7]. Using tumor-derived exosomes will provide additional competitive advantages for the selective delivery of anticancer therapies not only to the primary tumor but also to metastasis and even to the premetastatic niche, owing to their intrinsic organotropic tumor-homing properties $[8,9]$. Besides, tumorderived exosomes are also involved in a wide range of biological processes, including tumor progression, metastasis formation, and drug resistance, mechanisms 
that still remain poorly understood, making it necessary to generate a deeper knowledge before considering their safe application in therapeutics $[10,11]$.

Referring to the application of exosomes as cancer drug delivery systems, and besides the increasing interest overseen over the last years, only plant-derived exosomes loaded with curcumin for the treatment of colon cancer are currently under clinical evaluation with an ongoing phase I clinical trial (NCT01294072). This low translation rate might be related to the tedious and time-consuming isolation processes, as well to the lack of standardization regarding the production, characterization, and quality control assessment of the isolated exosomes. Safety concerns, due to their still unknown compositions and undescribed functionalities, severely hamper the clinical application of tumor-derived exosomes.

The potential of nanotechnology for developing improved anticancer therapies can be summarized in an increasing number of nanomedicines approved for the treatment of cancer [12]. However, the concept of the 'magic bullet', popularized by Paul Ehrlich at the beginning of the XX century, is still an ambitious objective in the field of nanotechnology, drug delivery, and cancer [1]. Nanostructures that resemble tumor-derived exosomes, both structurally and functionally, can provide a real alternative for the development of targeted anticancer therapies. It is generally accepted that exosomes are enriched in certain lipid species and share common groups of proteins, some of them responsible for their tumor-homing properties $[9,13,14]$. Therefore, by the combination of these materials and selection of an appropriate production methodology, it is possible to engineer EMNs.

We hence propose the development of EMNs, by integrating key components of tumor-derived exosomes, and provide evidence of their potential for the targeted delivery of anticancer therapeutics to the target site, while overcoming the main limitations of their natural counterparts.

\section{Results}

\section{Development and characterization of a nanoplatform structurally similar to exosomes}

Our purpose was to design a nanoplatform that resemble exosomes for their composition and physicochemical properties, of utility for the selective delivery of anticancer therapies. For reference, we isolated and characterized cell-derived extracellular vesicles (Additional file 1: Figure S1). WB analysis allowed confirming the effectiveness of the isolation protocol (Additional file 1: Figure S1a), and extensive proteomic analysis (LC-MS/MS) led to the identification of other 90 exosomal protein markers described in the top 100 proteins of the ExoCarta and
EV Vesiclepedia databases (Additional file 1: Figure S1b). Nearly $80 \%$ of the identified proteins were also classified as part of "exosome component" in the Gene Ontology Cellular Component section of the FunRich tool (Additional file 1: Figure S1c). Exosomes with characteristic cup-shape morphologies were observed by TEM (Additional file 1: Figure S1d), their yield of production determined (Additional file 1: Figure S1e) and their physicochemical properties measured by DLS and LDA (Table 1).

We next attempted the preparation of EMNs. We took into consideration previously published lipidomics works describing the most common lipid species enriched in tumor exosomes compared to parent cells, to define their composition (Additional file 1: Figure S2a) [13-15]. We optimized the preparation methodology based on previous knowledge using the ethanol injection method (we studied the influence of several parameters, lipid concentration, volumes, and the ratio of the components, in the physicochemical properties of the resulting liposomes), in order to obtain nanoplatforms structurally similar to exosomes in a single step (Additional file 1: Figure S2b). The properties of the obtained EMNs (schematically represented in Additional file 1: Figure S2c) are disclosed in Table 1. EMNs composition can be further modulated in order to obtain nanovesicles of different sizes and properties (Additional file 1: Table S1). Stability studies revealed great colloidal stability during storage (Fig. 1a), and upon incubation with cell culture media and human plasma (Fig. 1b). Characterization of 19 independent batches confirmed the excellent reproducibility of the formulation (Fig. 1c). NMR results $\left({ }^{1} \mathrm{H}\right.$ and ${ }^{31} \mathrm{P}$ NMR) prove that each lipid component was effectively incorporated into EMNs (Fig. 1d). While the spectrum corresponding to broken EMNs displayed relevant peaks corresponding to the free lipid molecules (according to the spectra of the pure components $\mathrm{PC}, \mathrm{SM}$, and $\mathrm{CH}$, colored rectangles),

Table 1 Physicochemical properties of exosomes and EMNs

\begin{tabular}{lccr}
\hline & Size $(\mathbf{n m})^{\mathbf{a}}$ & Pdl & ZP (mV) \\
\hline Exosomes from human plasma & $98 \pm 12$ & 0.4 & $-16 \pm 1$ \\
Exosomes from cancer cell lines & $91 \pm 11$ & 0.3 & $-23 \pm 2$ \\
EMNs & $100 \pm 8$ & 0.2 & $-7 \pm 2$ \\
F-EMNs & $110 \pm 2$ & 0.3 & $-6 \pm 1$ \\
EMNs + miR145 & $104 \pm 2$ & 0.3 & $-16 \pm 2$ \\
F-EMNs + miR145 & $113 \pm 1$ & 0.3 & $-5 \pm 2$ \\
\hline
\end{tabular}

Data presented as mean \pm standard deviation; $\mathrm{n}=3$

$P d l$ polydispersity index, ZP zeta potential, EMNs Exosome-mimetic nanosystems, F-EMNs functionalized EMNs with a specific integrin, miR145 microRNA-145, EMNs + miR145 EMNs loaded with miR145, F-EMNs + miR145 F-EMNs loaded with miR145

a Size corresponds to number measurement in DLS 

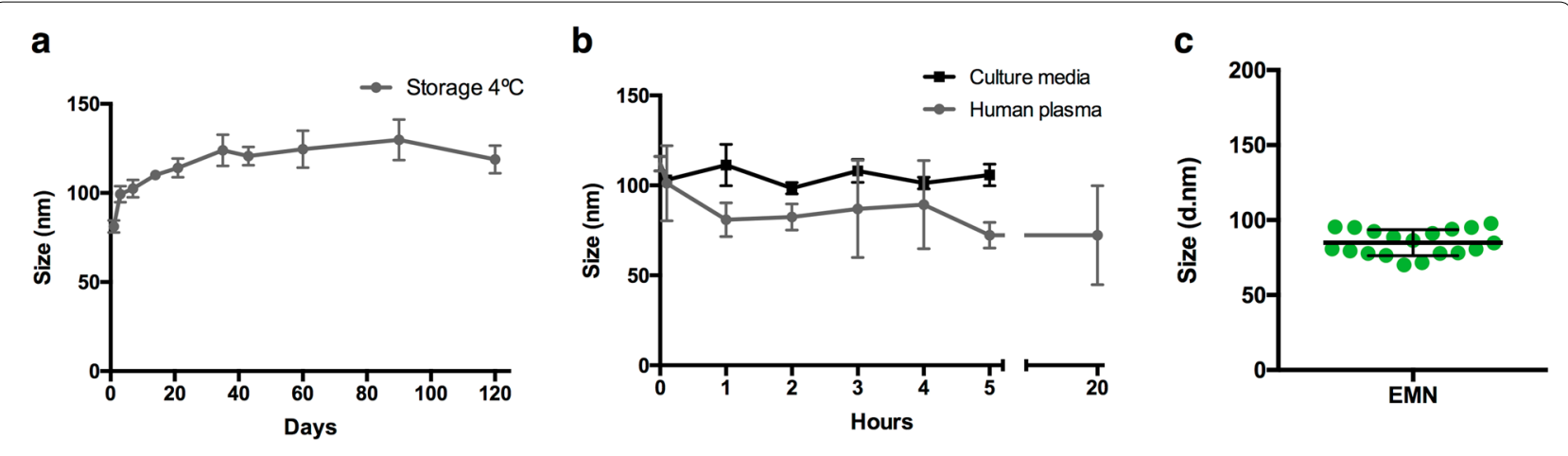

d

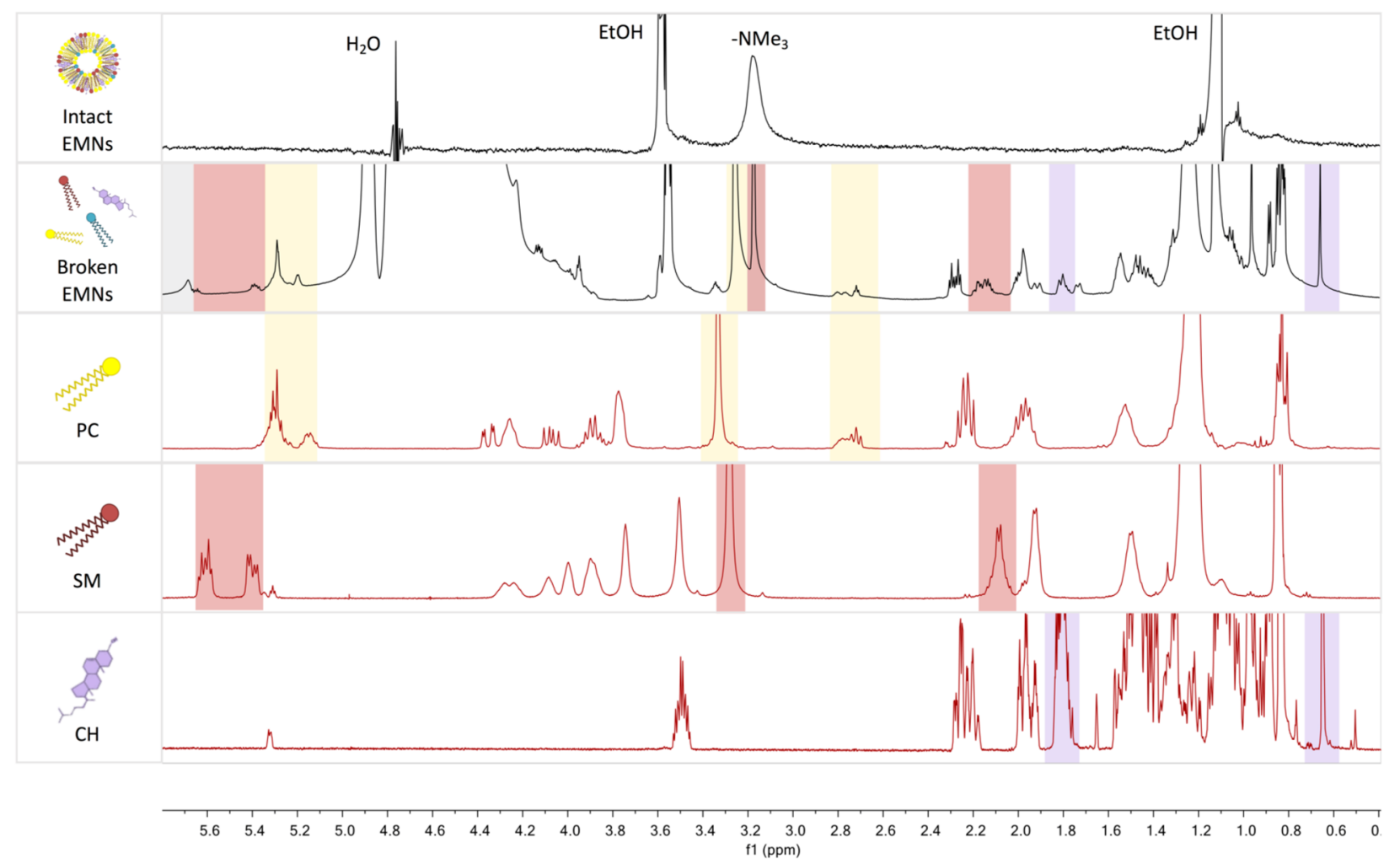

Fig. 1 Exosome-mimetic nanoplatforms characterization. a Hydrodynamic size (Z-average) of EMNs measured by dynamic light scattering (DLS) under storage conditions $\left(4^{\circ} \mathrm{C}\right.$ ) and $\mathbf{b}$ in culture media and human plasma, over the time (from t $=0$ to 120 days, $5 \mathrm{~h}$ and $20 \mathrm{~h}$, respectively; $\mathrm{n}=6$ ). $\mathbf{c}$ Hydrodynamic size measured by DLS of 19 independent batches of EMNs (green dots); horizontal bar represents mean $\pm S D$. $\mathbf{d}^{1} \mathrm{H}-\mathrm{NMR}$ spectrum showing representative signals of each component found in intact $\left(D_{2} O\right)$ and broken EMNs $(M e O D)$ compared to the spectrum of the pure components (PC, SM, and CH). Specific peaks of each component identified in the sample of broken EMNs were highlighted with colored rectangles ( $\mathrm{PC}$, yellow rectangles; $\mathrm{SM}$, red rectangles; $\mathrm{CH}$, purple rectangles)

the intact EMNs spectrum did not display practically any peak (only a terminal methyl group), meaning that free unreacted lipids were not present in the suspension. The ${ }^{31} \mathrm{P}$ NMR spectrum was also acquired for a double-checking, allowing to observe two peaks (broken EMNs) corresponding to the phosphorylated species of SM and PC. Moreover, this analysis resulted in a proportion of 0.48:1
(SM:PC), similar to the theoretical proportion 0.44:1, revealing that each lipid was efficiently and completely incorporated in EMNs in the exact proportion used for their preparation (Additional file 1: Figure S3).

We provide a methodology for preparation of EMNs structurally similar to exosomes (Table 1, Fig. 2a), while overcoming important limitations: (i) a single batch of 
a
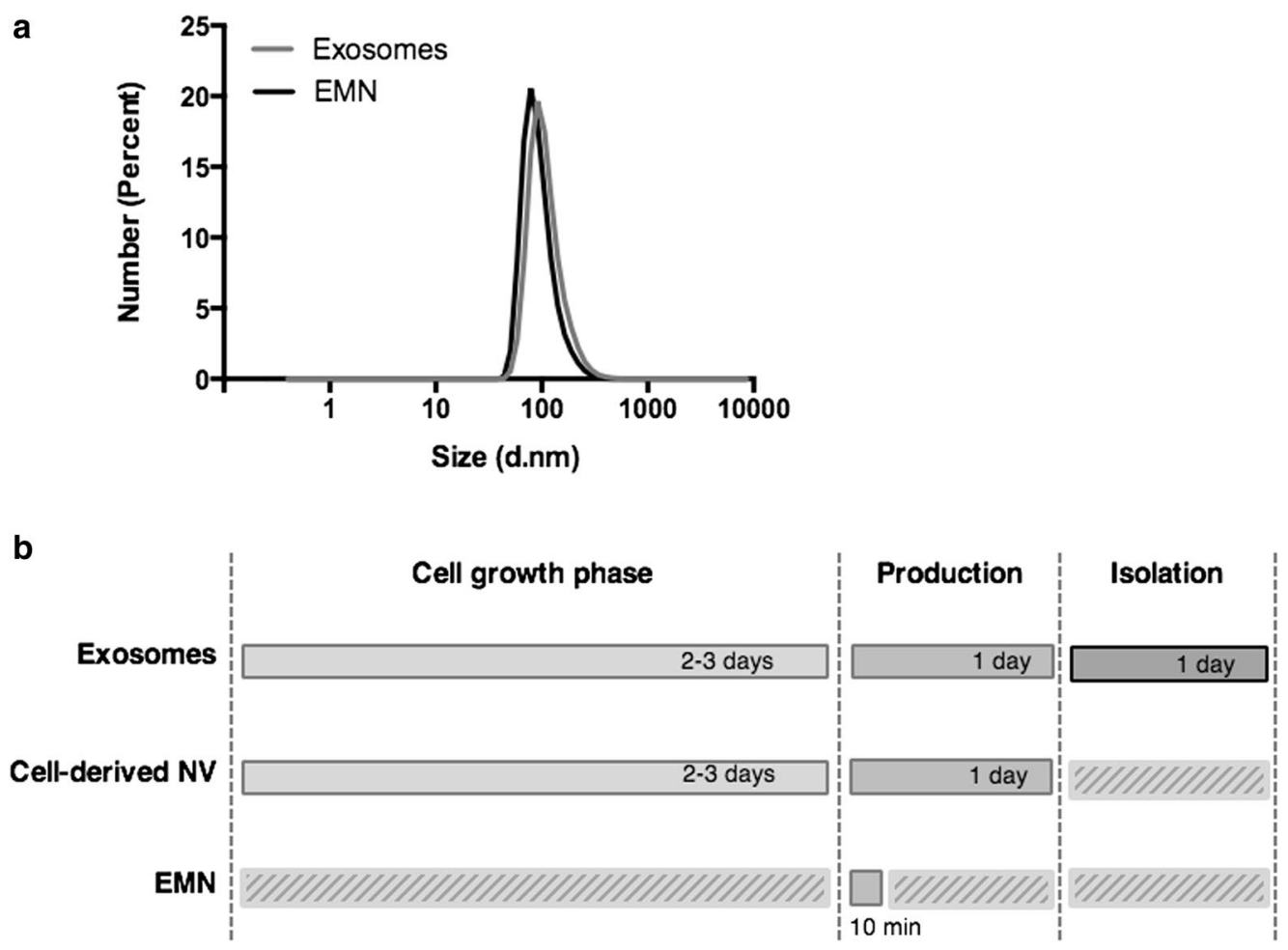

C

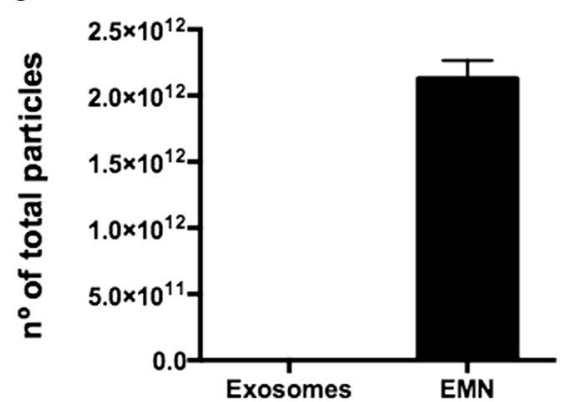

d

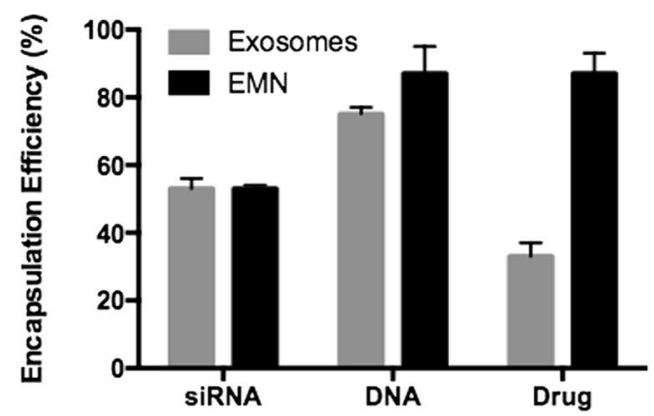

Fig. 2 Comparison between natural exosomes and EMNs. a Size distribution measured by DLS of natural exosomes and EMNs showing that we have obtained nanoplatforms of practically the same size than natural exosomes. $\mathbf{b}$ Time-consuming comparison for obtaining natural exosomes from cell lines and isolation by serial ultracentrifugation, cell-derived nanovesicles (NV) and EMNs. c The number of particles obtained in $216 \mathrm{ml}$ of conditioned medium ( $16 \mathrm{~h}$ ) of A549 exosomes and one batch of EMNs ( $\left.2^{\prime} 2 \mathrm{ml}\right)$. $\mathbf{d}$ Encapsulation efficiencies of therapeutic model molecules comparing the loading capacity of natural exosomes and EMNs. Bar charts represent mean \pm standard deviation, $n=3$

EMNs $\left(2^{\prime} 2 \mathrm{ml}\right)$, can be produced in $10 \mathrm{~min}$, while the exosome isolation time from conditioned medium by serial ultracentrifugation (Additional file 1: Figure S1e, protocol detailed in Additional file 1: Methods) takes several days, therefore offering a time-saving efficiency of at least 5 days for each production run; this production process is also faster than other current alternatives to exosomes, such as cell-derived nanovesicles by serial extrusion, which also requires time for cell growing and production [16] (Fig. 2b), (ii) the production yield, measured by the number of obtained particles, is 1000 -fold higher for EMNs than for exosomes (Fig. 2c), (iii) EMNs have a similar drug loading capacity than exosomes in the case of RNA (hydrophilic compound), and superior drug loading efficiencies in the case of DNA modified with a cholesterol chain (amphiphilic compound), and hydrophobic compounds such as curcumin (Fig. 2d), (iv) EMNs can be efficiently internalized by different cancer cells, and subsequently, deliver their payload intracellularly (Additional file 1: Figure S2d) without showing toxicity (Additional file 1: Figure S2e), (v) lipids are cheap and well-characterized materials, and finally and very importantly from a 
translational perspective, (vi) industrial production processes under GMP conditions are already well established for liposomes [17].

\section{Association of bioactive macromolecules}

We next increased the complexity of the formulation to include bioactive macromolecules, RNAs and proteins, to obtain EMNs with similar functionalities to simplified exosomes (Table 1). EMNs showed a good capacity to associate different types of proteins, irrespective of their MW and pI, without observation of significant changes in their physicochemical properties (nanoparticle size, distribution, and surface charge) and with association efficiencies over 80\% (Additional file 1: Table S2, Additional file 1: Figure S4). This is particularly important, allowing envisioning a versatile nanosystem that can be tailored with active proteins providing organotropic functionalities. Exosomal ITG $\alpha 6 \beta 4$, related with lung organotropism [9], was successfully bound to EMNs as verified by fluorescent WB (Fig. 3a shows a red signal in the loading well that corresponds to the protein bound to the EMNs), with the aim of increasing their targeting capability to the lung and enhancing their adhesive properties to recipient cells. The resulting EMNs functionalized with ITG $\alpha 6 \beta$ (F-EMNs), and labeled with NBD, had the capacity to mediate specific and effective interactions with laminin-5, according to a binding assay performed with laminin-5 coated plates. NBD-labeled plain EMNs were included in the experiment as the control formulation (Fig. 3b). Additionally, F-EMNs were perfused through a 3D culture model, previously developed by our group [18], in order to evaluate their interaction with A549 cells that express laminin-5 under dynamic conditions (Additional file 1: Figure S5a). Confocal images confirmed an efficient interaction of F-EMNs with the cells and show a good colocalization with laminin-5 (Additional file 1: Figure S5b).

Subsequent experiments were conducted to explore the potential of F-EMNs to transport RNAs to cancer cells. Besides cationic lipids are not present in the composition [19], we proved that EMNs efficiently associate therapeutic oncosuppressor miR145 by performing an agarose gel retention assay (Fig. 3c). Hence, we eventually engineered EMNs that simultaneously incorporate lipids, proteins, and RNAs, and resemble simplified exosomes with respect to their composition, physicochemical properties, and functionalities (Table 1). Confocal images proved that EMNs mediate an efficient delivery of miR145-Cy5 to A549 cells and support our notion that integrin functionalization indeed increases the adhesive properties in the case of F-EMNs as well as the transfection efficiency (Fig. 3d, e). Moreover, looking at the $3 \mathrm{D}$ reconstruction of the confocal images (Additional file 1: Figure S4c) and $\mathrm{xz}$ and yz-slices (Additional file 1: Figure S4d), a clear colocalization of NBD-labeled F-EMNs and miR145-Cy5 was observed, proving that the payload and the carrier are traveling together. Also, EMNs provide great opportunities for being further amendable to add different fluorophores for broader applications (Additional file 1: Figure S6 depicts triple labeling of EMNs with TopFluor-SM, Cy5 and DiR). RT-PCR assays confirmed the superior behavior of F-EMNs for delivery of therapeutic RNAs to cancer cells, which rendered a fivefold increase in the expression of miR145 with respect to the control formulation without ITG $\alpha 6 \beta 4$ (EMNs + miR145) (Fig. 3f). As expected, control cells transfected with miR145 in solution (miR145 free) or with EMNs loaded with a scrambled sequence (EMNs + miRscr) did not show differences in the expression of miR145 compared to untreated cells. This increase in the intracellular levels of miR145 produced by F-EMNs was translated into a significant reduction of the clonogenic capacity of the transfected cells (Additional file 1: Figure S7a). Additionally, to evaluate whether F-EMNs could be trapped in the endolysosomal degradation pathway [20], a lysosomotropic agent that induces lysosomal membrane perturbation, called chloroquine [21, 22], was added to the cells previously transfected. Results of a colony formation assay showed that no significant differences were observed when chloroquine was added, proving that F-EMNs can efficiently escape the endolysosomal

\footnotetext{
(See figure on next page.)

Fig. 3 Functionalization of EMNs with ITGa6ß4 (F-EMNs) and delivery of miRNA145. a Fluorescent Western blot showing the effective association of ITGa6ß4 with EMNs (F-EMNs) by ultracentrifugation at 120,000 $\times g$ (pellet) compared to the supernatant (SN). b Specific interaction of F-EMNs to coverslips coated with laminin-5. EMNs and F-EMNs were labeled with the fluorophore NBD-CH (green). Scale bars represent $100 \mu \mathrm{m}$. c Gel retention assay showing the free microRNA145 compared to the miR145 encapsulated in EMNs that remains stacked in the well of the gel. $\mathbf{d}$ Confocal microscopy images after $4 \mathrm{~h}$ transfection of A549 cells with EMNs + miR145 and F-EMNs + miR145. Blue channel: nuclei (DAPI); green channel: EMNs and F-EMNs (NBD-CH); red channel: miR145 (Cy5). Scale bars represent $25 \mu \mathrm{m}$. e FACS quantitative analysis of the transfection efficiency of F-EMNs + miR145-Cy5 compared to EMNs + miR145-Cy5, and the control cells. f Real time-qPCR of miR145 levels in A549 cells after $4 \mathrm{~h}$ transfection with miR145 free, a scramble sequence, EMNs + miR145 and F-EMNs + miR145. Data are representative of three independent experiments
} 


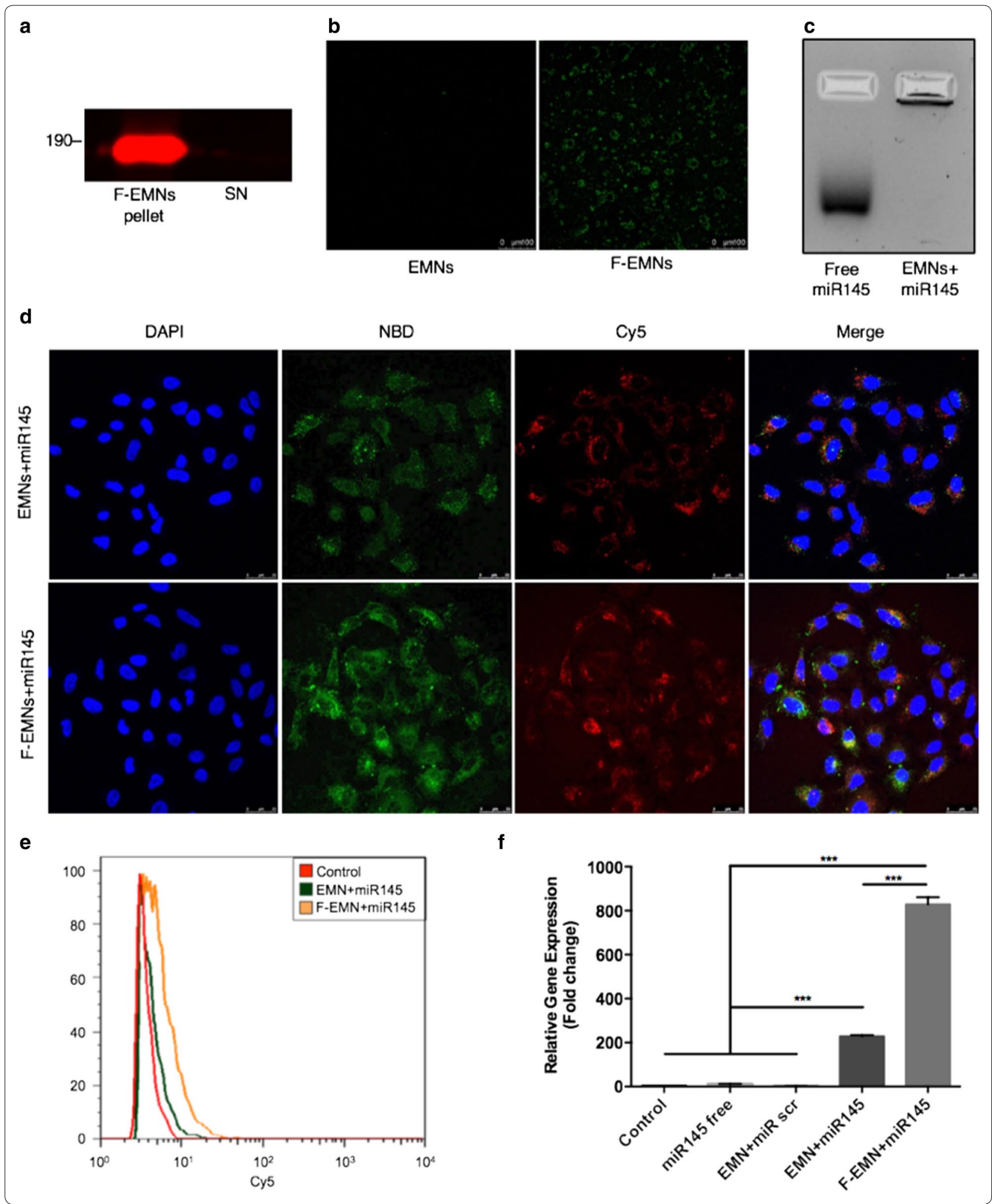


system (Additional file 1: Figure S7b). Moreover, at the protein level, F-EMNs loaded with miR145 significantly reduced the expression of $\mathrm{N}$-cadherin in the transfected cells, a protein that promotes tumor cell survival, migration, and invasion, and one of the targets of miR145 in lung adenocarcinoma [23] (Additional file 1: Figure S7c). Therefore, F-EMNs are able to deliver functional miR145 to cancer cells, overcoming the main barriers in gene delivery.

\section{F-EMNs have a similar capacity than tumor-derived exosomes to transport therapeutic RNAs to cancer cells}

Final experiments were established to compare tumorderived exosomes with engineered F-EMNs loaded with miR145, both represented in Fig. 4a. Cryo-TEM images revealed similarities with respect to the vesicle size, spherical shape, and membrane thickness (Fig. 4b). Transfection experiments in vitro proved that miR145loaded F-EMNs are able to transport genetic material to tumor A549 cells in a comparable fashion to exosomes isolated from the same cell culture, as it can be seen by confocal microscopy pictures and FACS analysis (Fig. 4c, Additional file 1: Figure S8). Next, in vivo biodistribution experiments in mice bearing lung cancers (inoculation of luciferase-expressing A549 lung carcinoma cells into the tail vein of nude mice leading to tumor formation) show a comparable behavior of F-EMNs and tumorderived exosomes both loaded with miR145-Cy5. First experiments were conducted to determine the most suitable administration route. miR145-Cy5-loaded F-EMNs were administered either intraperitoneally (IP) or retro-orbitally (RO), since by these routes, lungs are not reached directly after injection. Results prove that F-EMNs + miR145-Cy5 could efficiently reach the tumors irrespective of the administration route (Additional file 1: Figure S9a), but a higher fluorescent signal in the liver, kidney, and spleen, was observed after RO in comparison to IP injection, indicative of a higher accumulation in these organs following the first administration route (Additional file 1: Figure S9b). Importantly, we did not observe any signal in the heart, supporting the idea that F-EMNs treatment would avoid cardiotoxicity, one of the main concerns related to the development of cancer therapeutics [24]. According to these results, we decided to pursue with the IP modality, which also proved to be adequate for administration of other types of nanocarriers loaded with biomolecules [25]. We injected miR145-Cy5-loaded F-EMNs and
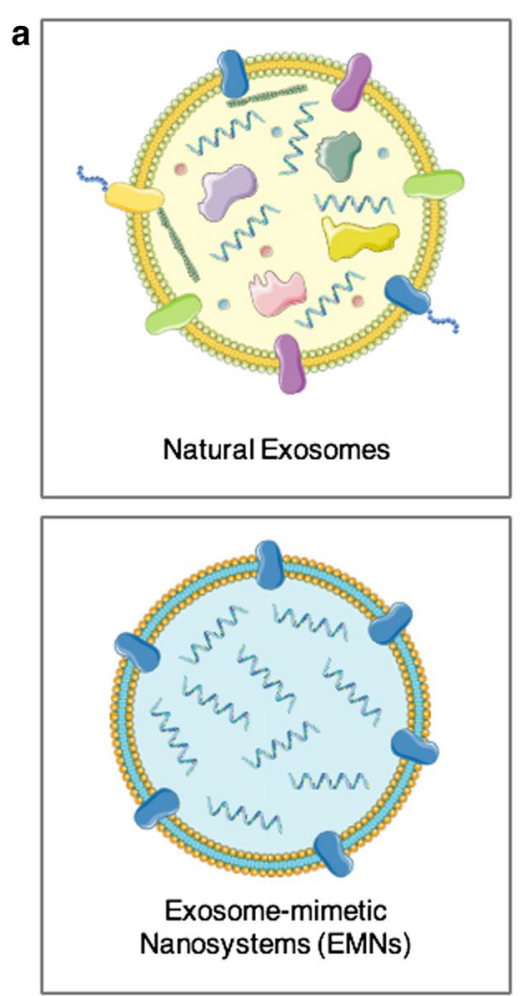

b
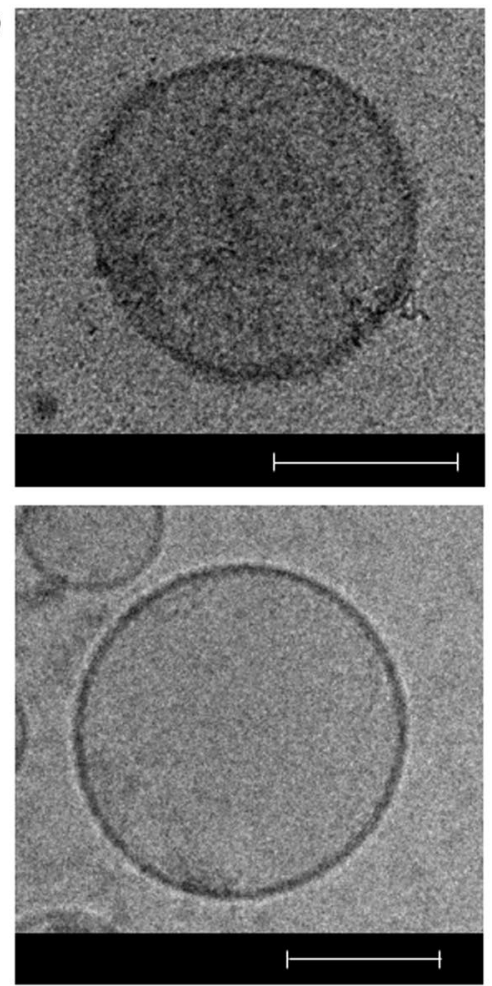

c
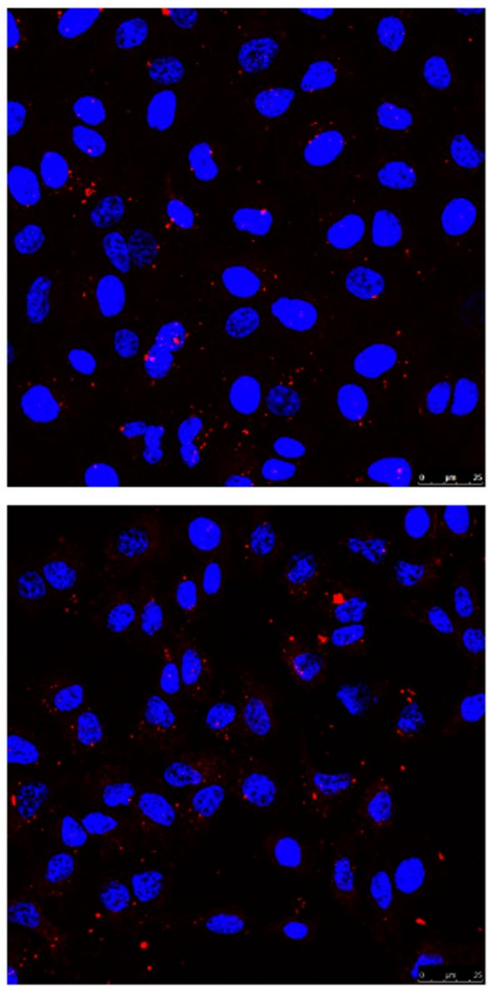

Fig. 4 Comparison of natural exosomes and EMNs morphology and miR145 association. a Schematic representation of the composition and morphology of natural exosomes (upper) and functionalized miR145-loaded EMNs (lower). b Cryo-TEM images of natural exosomes loaded with miR145 (upper) and F-EMNs + miR145 (lower). Scale bar represents $100 \mathrm{~nm}$. c Confocal images of miR145 delivery by natural exosomes (upper) and F-EMNs (lower) in A549 cells. Blue channel: nuclei (Hoechst); red channel: microRNA145 (Cy5). Scale bars represent $25 \mu m$ 


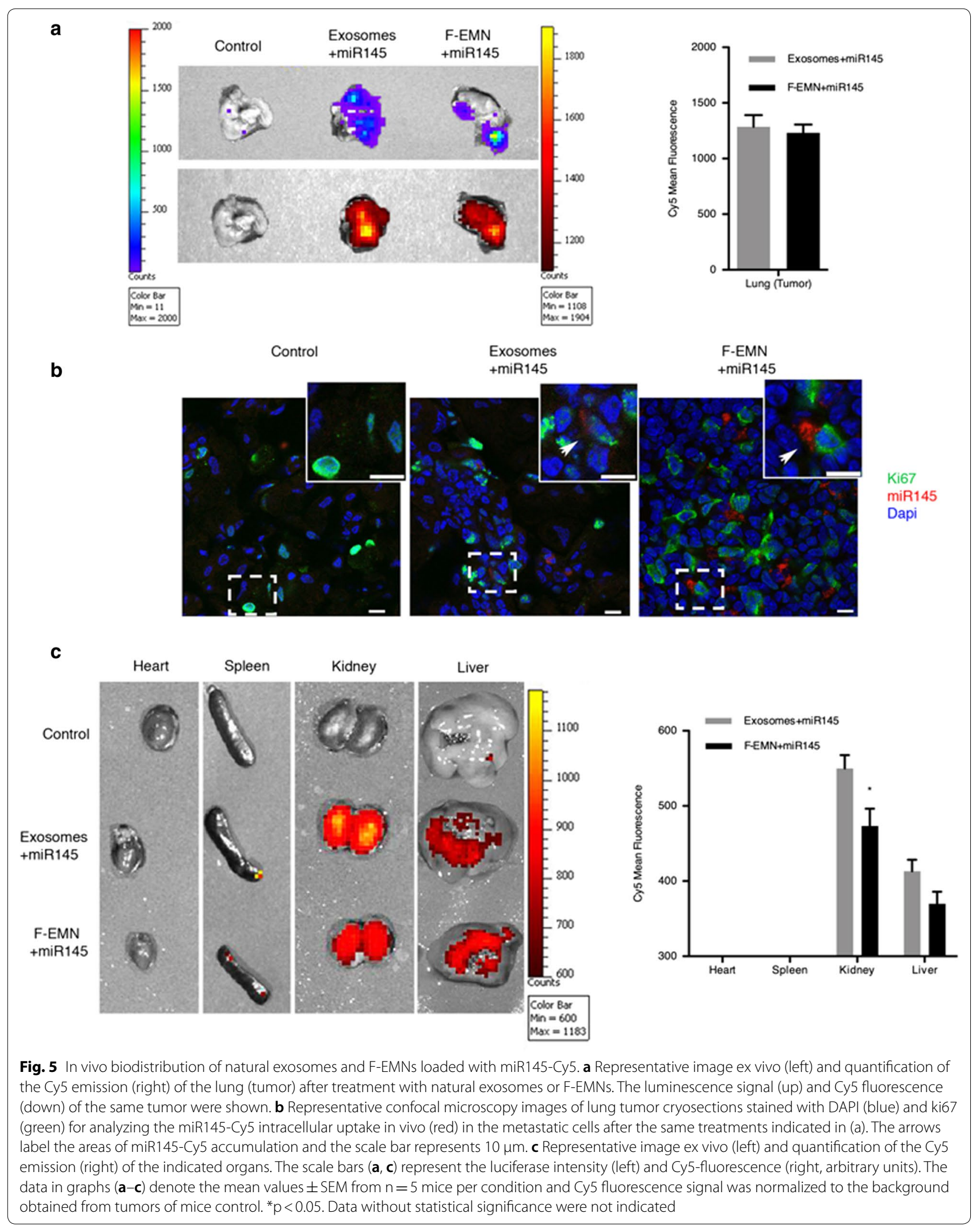


miR145-Cy5-loaded tumor-derived exosomes and confirmed that both treatments could mediate the accumulation of miRNA in the lung and the tumor with similar efficiency (Fig. 5a). Immunofluorescent studies with the excised tissue revealed localization of miR145-Cy5 in the neighborhood of Ki67 positive proliferative tumor cells (Fig. 5b). In addition, there was a significant reduction in the fluorescence signal observed in the kidney and the liver for mice receiving F-EMNs + miR145-Cy5 with respect to mice receiving exosomes, suggesting that F-EMNs can reach to the tumor efficiently, and probably provide lower systemic toxicities (Fig. 5c).

\section{Discussion}

Given their role in intercellular communication, exosomes are being increasingly explored as delivery systems for biomedical purposes. The organotropism described for tumor-derived exosomes to the tumor site and premetastatic niche could hold the key for designing highly efficient anticancer therapies targeting metastatic cancer with minimum side effects $[9,26]$. However, the main drawback for the application of this approach is the lack of more in-depth understanding of their molecular composition and function, raising safety concerns that must be resolved prior to promote their therapeutic application [10]. Moreover, the low yield, highly laborious, costly, and time-consuming methods of production for cell-derived exosomes, together with a lack of standardization for relevant processes such are determining their physicochemical properties and drug loading capacity, are additional challenges to overcome [27-29]. Researchers have tried to overcome some of these challenges by pursuing different strategies such as the generation of nanovesicles by serial cell extrusion, or the use of cell membranes (e.g. from erythrocytes, platelets, mesenchymal cells-MSC) for the coating of polymeric nanoparticles, rendering what they named exosome-mimics or synthetic exosomes [30-34]. However, these approaches do not overcome the challenges related to off-target signaling from proteins present in the vesicle surface, or the undesirable delivery of additional species present in the lumen [29].

In an attempt to overcome technical limitations and regulatory issues related to the clinical use of exosomes in cancer drug delivery, we have pursued a rational design of EMNs based on the well-known liposome technology. Liposomes are the most widely studied types of nanosystems and have successfully been translated into clinical products [17]. Therefore, EMNs can benefit for the accumulated knowledge in industrial scaling-up and GMP production of liposomes. Moreover, considering that liposomes are typically used as controls for preclinical evaluation of exosomes as drug delivery carriers, rational-designed EMNs can also have an application in this regard. Indeed, a recent review by Johnsen et al. highlights the crucial need for an adequate choice of liposomal controls in preclinical exosome-based drug delivery studies before postulate any potential superiority of exosomes over their liposomal counterparts [35]. Authors often choose over-simplified liposomes for their comparisons and rarely would choose a clinically relevant liposomal formulation. Gold standard liposome controls are urgently needed for a much fairer comparison.

EMNs provide additional opportunities of being further amenable to surface functionalization as well as loading of therapeutic cargo for a broader application in other fields. From our perspective, rather than just for competitive purposes, our nanoplatform could also serve as a tool for a deeper understanding of the still not answer questions of exosomes, for instance, what is the role of specific lipid species or proteins in the membrane surface of exosomes in trafficking, cellular uptake, or cell-to-cell interaction processes. The answers to these questions would benefit both fields for reaching sooner a novel clinically relevant drug delivery system.

Taking into account all these considerations, we have successfully engineered EMNs, mimicking simplified natural exosomes not only structurally, but also holding specific molecular features and functionalities (F-EMNs + miR145). EMNs are prepared in very mild conditions (e.g. avoiding $\mathrm{pH}$ changes and high-energy processes), using an optimized methodology that is fast, simple, and reproducible. Using the ethanol injection methodology, we are able to produce small nanoplatforms in few minutes without needing additional steps for adapting the size and lamellarity (e.g. extrusion or sonication) and keeping the amount of ethanol in the formulation below $10 \%$, meaning that it does not need to be removed prior to in vivo administration [36]. This preparation method resulted in a substantially increased production yield and reduced preparation time with respect to isolated tumor-derived exosomes. Moreover, these nanoplatforms are only composed of natural lipids enriched in exosomes [13]. Their use has been widely reported in other clinical formulations, therefore having a well-known safety profile. Importantly, the lipidic composition can be easily tailored, allowing incorporation of additional species that could be described in future for having relevant roles in trafficking, cell communication, or cell-to-cell interaction processes, accompanying advances in the lipidomic field [15, 37]. Lastly, EMNs efficiently incorporate labile macromolecules (i.e. RNA and proteins). Encapsulation of therapeutic proteins into liposomes has been attempted with great success in the case of insulin, calcitonin, VIP, and interleukins [38]. In our hands, we successfully functionalized 
our nanoplatforms with the extracellular fraction of the transmembrane protein integrin $\alpha 6 \beta 4$, that can be found in tumor-derived exosomes conferring tumor homing properties and proved that its presence improves the adhesion properties of EMNs and ability to deliver the associated therapeutics to cancer cells. EMNs can also associate miRNAs with comparable efficiency to exosomes, besides they are neutrally charged. Typically, nanocarriers for gene delivery incorporate cationic compounds bearing intrinsic toxicity [39], but neutral ones have recently been claimed a much safer alternative avoiding off-target effects [40]. Transfection studies show that our nanoplatforms functionalized with integrins, F-EMNs, can mediate an increase in the intracellular levels of miR145 of over 800-fold higher than the free miR145, and fivefold higher than the control formulation (EMNs). This increased expression related to relevant changes in cancer cells phenotype. In vivo experiments carried out in a lung cancer mice model allowed us confirming that F-EMNs show a similar capacity to transport their therapeutic cargo (miR145) to the target as compared to tumor-derived exosomes. Importantly, F-EMNs do not apparently provide cardiotoxicity and show a lower accumulation by the liver and kidney than their natural counterparts.

\section{Conclusions}

Overall, we have designed a multifunctional nanoplatform mimicking exosomes, F-EMNs loaded with RNAs, that can be manufactured and characterized in a controlled manner for a safer biomedical approach, and have demonstrated to efficiently transport bioactive macromolecules to the target cells in a similar fashion to tumorderived exosomes. We provide the first proof-of-concept of the potential of this technological nanoplatform as a real alternative to exosomes for the development of safer and more efficient anticancer therapies, a technology that is versatile and can be adapted as we go deep in the study of exosomes and the molecular features related to their tumor-homing properties.

\section{Methods}

\section{Materials}

Phosphatidylcholine (Lipoid E PC) and sphingomyelin (Lipoid E SM) were obtained from Lipoid GmbH (Ludwigshafen, Germany). Cholesterol was purchased from Sigma-Aldrich (Madrid, Spain). C16 Ceramide, NDB-6 Cholesterol, and C11 TopFluor Sphingomyelin, were all purchased in Avanti Polar Lipids (Alabaster, AL, USA). MilliQ ${ }^{\circledR}$ water (Simplicity 185, Millipore, Bedford, USA) was used throughout the study. Ethanol of analytical grade was purchased from VWR (Barcelona, Spain). DiR (DiIC18(7) (1,1'-Dioctadecyl-3,3,3', $3^{\prime}$-Tetramethylindotricarbocyanine
Iodide) and DiD (1, $1^{\prime}$-dioctadecyl-3,3,3,, $3^{\prime}$ tetramethylindotricarbocyanine perchlorate) were acquired from Thermo Fisher Scientific (USA). miRNA145 (miR145; sense strand 5'-GUCCAGUUUUCCCAG GAAUCCCU- ${ }^{\prime}$, antisense strand $5^{\prime}$-GGAUUCCUGGAA AUACUGUUCU-3'), miRNA-145-Cy5 (miR145-Cy5,), miRNA-scramble (miRscr) and a model siRNA were synthesized by Eurofins Genomics (Ebersberg, Germany). DNA-CH was kindly provided from Ramon Eritja (Nucleic Acids Chemistry Group, Institute for Advanced Chemistry of Catalonia, Barcelona, Spain).

\section{Cell culture}

SW480 (ATCC ${ }^{\circledR}$ CCL-228), PC-3 ATCC $^{\circledR}$ CRL-1435) and A549 (ATCC $^{\circledR}$ CCL-185) cells were grown in Dulbecco's modified Eagle's medium (DMEM) high glucose (Gibco, Thermo Fisher Scientific), supplemented with $10 \%$ fetal bovine serum (FBS) (Thermo Scientific, Spain), and $1 \%$ penicillin/streptomycin (Thermo Scientific, Spain). Cells were maintained at $37{ }^{\circ} \mathrm{C}$ in a $5 \% \mathrm{CO}_{2}$ humidified atmosphere. Trypsin and Phosphate Buffered Saline (PBS) were purchased from Sigma-Aldrich (St. Louis, USA). All cell lines were tested routinely and confirmed to be mycoplasma-free. The A549 cells were authenticated by STR-profiling according to ATCC guidelines.

\section{EMNs preparation and characterization Preparation of EMNs}

EMNs with a well-defined composition (CH:PC:SM:Cer) were prepared following the ethanol injection methodology. In brief, lipids were dissolved in ethanol, at defined ratios (0.9:1:0.4:0.03 w/w), and a total lipid concentration of $0.92 \mathrm{mg} / \mathrm{ml} .200 \mu \mathrm{l}$ of the ethanol solution was injected with an insulin syringe $(0.5 \mathrm{ml}, 0.33 \times 12 \mathrm{~mm}$ ICO.C.1) into $2 \mathrm{ml}$ of milliQ water, under magnetic stirring. EMNs were spontaneously formed.

\section{Dynamic light scattering (DLS) and laser Doppler anemometry (LDA)}

The hydrodynamic diameter, polydispersity index and superficial charge of the exosomes and EMNs were measured using a Zetasizer Nano ZS (Malvern Instruments, UK). Measurements were performed in PBS 1X (exosomes) and MilliQ water (EMNs) at room temperature (RT). For the zeta potential measurements, samples were diluted in $1 \mathrm{mM}$ potassium chloride $(\mathrm{KCl})$.

\section{Nanoparticle tracking analysis (NTA)}

Particle size and concentration distribution of the EMNs and exosomes were also measured using NTA (v2.3; Malvern Instruments, Malvern, UK) according to manufacturer's instructions. Briefly, EMNs samples were vortexed 
and diluted to a final dilution of 1:1000 in milliQ $\mathrm{H} 2 \mathrm{O}$ and exosomes 1:100. Blank-filtered $\mathrm{H}_{2} \mathrm{O}$ was run as a negative control. Each sample analysis was conducted for $60 \mathrm{~s}$ and measured five times using Nanosight automatic analysis settings. The detection threshold was set to level 11 and camera level to 15 .

\section{Stability}

A stability study was performed in human plasma and cell culture media (DMEM supplemented with 1\% FBS) incubated at $37{ }^{\circ} \mathrm{C}$. The colloidal properties of EMNs were determined using the Zetasizer Nano ZS each hour up to $5 \mathrm{~h}$ for human plasma and $20 \mathrm{~h}$ for DMEM. Stability of the formulation under storage conditions was also tested in PBS $1 \mathrm{X}$ at $4{ }^{\circ} \mathrm{C}$ up to 3 months. All measurements were performed in sextuples.

\section{Nuclear magnetic resonance spectroscopy (NMR)}

NMR experiments relying on ${ }^{1} \mathrm{H}$ and ${ }^{31} \mathrm{P}$ detection were measured on a Varian Inova $17.6 \mathrm{~T}$ spectrometer (750 MHz proton resonance) equipped with a triple resonance $\mathrm{HCP}$ probe and z-gradient. The ${ }^{13} \mathrm{C}$ NMR spectra were measured on a Varian Mercury 7.04 T (75.4 MHz, ${ }^{13} \mathrm{C}$ resonance) equipped with a double resonance ATB probe with z-gradient. The spectra were processed and analyzed with MestreNova software v11.0 (Mestrelab. inc.).

\section{Cryogenic transmission electron microscopy (cryo-TEM)}

Samples were initially vitrified according to Dubochet protocol [41]. Briefly, an aliquot of $3.5 \mu \mathrm{l}$ of each sample was applied to glow-discharged holey grids for $1 \mathrm{~min}$, blotted, and rapidly plunged into liquid ethane at $-180^{\circ} \mathrm{C}$ and kept at this temperature until visualization. Images were obtained at $0^{\circ}$-tilt under minimum dose conditions using a field emission gun Tecnai 20 G2 Microscope (FEI, Eindhoven, The Netherlands) equipped with a Gatan cold stage operated at $200 \mathrm{keV}$. Low-dose images were collected at a nominal magnification of $\sim 50,000 \times$ by using an FEI Eagle CCD camera with a step size of $15 \mu \mathrm{m}$. The original pixel size of the acquired images was $2.74 \AA$.

\section{Loading of therapeutic molecules Exosomes}

Exosomes were loaded with different molecules. The hydrophobic drug Curcumin (Acros Organics ${ }^{\mathrm{TM}}$, ThermoFisher Scientific) was loaded into exosomes ( $0.5 \%$ loading $\mathrm{w} / \mathrm{w}$ ) by incubation for $10 \mathrm{~min}$ in the dark. Curcuminexosomes were then isolated by ultracentrifugation at $120,000 \times g$ for $1 \mathrm{~h}$ at $15^{\circ} \mathrm{C}$ in an SW32 Ti rotor (Optima TL Ultracentrifuge, Beckman Coulter) and resuspended in MilliQ water. Exosomes were also loaded with nucleic acids (random siRNA, DNA-CH or miRNA145) by electroporation as previously described [42]. Briefly, exosome pellet was resuspended in PBS 0.1X and gently mixed with the appropriated $\mu$ of siRNA, DNA-CH or miRNA (same loading than for EMNs) in a final volume of $400 \mu \mathrm{l}$ into $0.4 \mathrm{~cm}$ electroporation cuvettes. Exosomes were then electroporated using a Gene Pulser II Electroporator (Bio-Rad), at $300 \mathrm{~V}$ and $25 \mu \mathrm{F}$ of capacitance. Lastly, exosomes were incubated in ice for $30 \mathrm{~min}$ to allow the exosome membrane to be fully restored. To get rid of free nucleic acids, exosomes were diluted with cold PBS and isolated again by ultracentrifugation at $120,000 \times g$ for $90 \mathrm{~min}$ at $15{ }^{\circ} \mathrm{C}$ in $70.1 \mathrm{Ti}$ rotor (Optima TL Ultracentrifuge).

\section{EMNs}

Loading with curcumin was accomplished by adding the drug $(0.5 \%$ loading $\mathrm{w} / \mathrm{w})$ to the ethanolic lipidic phase prior injection into the aqueous phase. The suspension was then kept under stirring for $10 \mathrm{~min}$ in the dark at $\mathrm{RT}$ and ultracentrifuged at $35,000 \mathrm{rpm}$ for $1 \mathrm{~h}$ at $15^{\circ} \mathrm{C}$. To associate siRNA and dsDNA to EMNs, the required amount of each nucleic acid was dried up (to eliminate the water content) using miVac DUP with Quattro pump (Genevac) and the organic phase was added, vortexed at $12 \mathrm{rpm}$ for $1 \mathrm{~min}$ and injected in the aqueous phase together with the lipids. miR145 and miR-scramble $(8 \mu \mathrm{g})$ was associated with EMNs by adding directly $8 \mu \mathrm{g}$ of miRNA in the organic phase and injecting it in the aqueous phase. The formulation was kept 10 min under magnetic stirring.

Encapsulation efficiencies (\%EE) of the drug and nucleic acids in the nanovesicles were determined indirectly by the difference between the total amount of the theoretical amount added in the sample and the free amount found in the supernatant after isolation by ultracentrifugation. The free drug was detected by measuring the fluorescence curcumin emits $(\lambda E x=420 \mathrm{~nm}$, $\lambda \mathrm{Em}=535 \mathrm{~nm})$. Free nucleic acids were detected by using the SYBER Gold solution $(\lambda E x=500 \mathrm{~nm}, \lambda E \mathrm{E}=550 \mathrm{~nm})$ and the \%EE was calculated following the next equation:

$$
\% \mathrm{EE}=(\mathrm{W} \text { theoretic }-\mathrm{W} \text { free }) / \mathrm{W} \text { theoretic } \times 100 .
$$

Semi-quantification of miR145 encapsulated in EMNs was performed by agarose gel retention assay. Briefly, EMNs + miR145 were concentrated 10 times by a rotavapor and loaded onto a $2 \%$ agarose gel in TAE $1 \mathrm{X}$ buffer. Electrophoresis was performed at $100 \mathrm{~V}$ for $40 \mathrm{~min}$. MiR145 was visualized by SYBR ${ }^{\circledR}$ Gold Nucleic Acid Gel Stain (Invitrogen) by UV transillumination and gel photography. Free miR145 was used as a control. 


\section{EMNs functionalization with proteins}

EMNs were functionalized with different proteins, recombinant human integrin $\alpha 6 \beta 4$ protein (Vitro), Bovine Serum Albumin (BSA, VWR) and Lysozyme (Sigma-Aldrich) by incubation. Briefly, EMNs were prepared as described above, and proteins were added to the formulation at a ratio 1:100 (protein:lipids) on an orbital shaker for $20 \mathrm{~min}$ at RT. To assess the protein association of ITG $\alpha 6 \beta 4$, F-EMNs were ultracentrifuged at $60,000 \mathrm{rpm} 1 \mathrm{~h} 15^{\circ} \mathrm{C}$ in a Beckman 70.1 Ti rotor (Optima TL Ultracentrifuge) to isolate functionalized EMNs from free protein, and both fractions were loaded on $10 \%$ acrylamide gel and detected by fluorescent western blotting using the primary monoclonal antibody ITG $\beta 4$ (G-7, Santa Cruz Biotechnology). Physicochemical properties of functionalized EMNs were also measured by DLS and LDA. EMNs + miRNA145 were functionalized with ITG $\beta 4$ following the same procedure.

\section{Specific interaction integrin-laminin}

Coverslips were coated with $1 \mathrm{ml}(10 \mu \mathrm{g} / \mathrm{ml})$ of Laminin$\mathrm{V}$ (Cultrex ${ }^{\circledR}$, Vitro) and incubated at $37^{\circ} \mathrm{C} \mathrm{o} / \mathrm{n}$. Coverslips were then washed twice with PBS $1 \mathrm{X}$ and $100 \mu \mathrm{l}$ of EMNs with/without integrin functionalization (EMNs and F-EMNs) were added and incubated for $24 \mathrm{~h}$ at $37{ }^{\circ} \mathrm{C}$. Then, the coverslips were washed again $3 \times 5$ min with PBS $1 \mathrm{X}$ and mounted on slides with $8 \mu \mathrm{l}$ of Mowiol 4-88 Reagent (Merck, Spain), dried at RT and conserved in the dark at $-20{ }^{\circ} \mathrm{C}$ to be analyzed later on by the confocal microscopy.

\section{miR145 transfection}

\section{Confocal microscopy analysis}

A549 cells were seeded on coverslips in 24-well plate at a density of $8 \times 10^{4}$ cells/well in complete medium. The following day, the same quantity of EMNs or F-EMNs labeled with NBD-CH and loaded with miR145-Cy5 were incubated with the cells in medium without supplement for $4 \mathrm{~h}$ in the dark. After incubation, medium was removed, cells were washed with PBS three times and then, fixed with paraformaldehyde (PFA; $4 \% \mathrm{v} / \mathrm{v}$ in PBS) in the dark at RT for 15 min prior to counterstain the nuclei with DAPI for $10 \mathrm{~min}$. Lastly, coverslips were mounted on clean slides with $8 \mu \mathrm{l}$ of Mowiol, dried at RT, and conserved in the dark at $-20{ }^{\circ} \mathrm{C}$ to be analyzed later on by Confocal LaserScanning Microscope (CSLM, Leica TCS SP5). Cells were also transfected with miR145-Cy5-loaded exosomes and F-EMNs, adding the same amount of miR145-Cy5 $(8 \mu \mathrm{g})$ to the cells and following the just mentioned protocol.

\section{FACS analysis}

miR145-Cy5-loaded EMNs, F-EMNs and exosomes were incubated with the cells as described above.
After incubation, the medium was removed, cells were washed with PBS three times and trypsinized. A549 cells incubated with PBS instead of nanovesicles were also included as a control. Collected cells were fixed in $0.4 \%$ paraformaldehyde and kept at $4{ }^{\circ} \mathrm{C}$ until FACS analysis. The percent of $\mathrm{Cy} 5$ positive cells was determined by a FACScan flow cytometer (BD Biosciences). A minimum of 10,000 events per condition was measured. The analysis of the results was performed using FlowJo Software (TreeStar Inc., Ashland, USA).

\section{Transfection}

$24 \mathrm{~h}$ before transfection, A549 cells were seeded on 6 -well plate at a low density of $25 \times 10^{4}$ cells/well in complete DMEM. The next day, complete medium was removed, cells were washed with PBS $1 \mathrm{X}$ and new medium without supplement was added. $8 \mu \mathrm{g}$ of miR145 associated with EMNs and F-EMNs, as well as miR145 free in solution (as a control) were added to each well and incubated at $37{ }^{\circ} \mathrm{C}$ and $5 \% \mathrm{CO}_{2}$ atmosphere for $4 \mathrm{~h}$. After incubation, the culture medium was removed, cells were washed with PBS and fresh complete medium was added and further incubated for $96 \mathrm{~h}$ prior to being collected for analyses by RT-PCR and functional assays (colony forming assay and Western blot).

\section{RT-PCR analysis}

Total microRNA was extracted from transfected A549 cells using the microRNA Purification Kit (Norgen Biotek Corp.) following the manufacturer's protocol. After nanodrop RNA quantification, the RNA was retrotranscribed into cDNA using the $\mathrm{qScript}^{\mathrm{TM}}$ microRNA cDNA Synthesis Kit (Quanta Bioscience ${ }^{\mathrm{TM}}$ ) according to manufacturer's instructions. Quantitative Real TimePCR was performed using PerfeCta SYBR Green SuperMix (Quanta Bioscience ${ }^{\mathrm{TM}}$ ) in an AriaMx Real-time PCR System (Agilent Genomics). The relative quantities of miR145 were normalized using the housekeeping RNU-6 and using the comparative CT method. For miRNA quantitation, specific forward, reverse and universal primers were acquired from Eurofins (Fisher Scientific): hsa-miR145-5p (5'-CGCGCGTTCCAGTTTTCC CAGG-3') and universal reverse PCR primer (5'-GTG CAGGGTCCGAGGT-3'), and the housekeeping small RNA control primer RNU6 (5'-CTCGCTTCGGCA GCACA-3', 5'-AACGCTTCACGAATTTGCGT-3'). The PCR conditions consisted of 2 min of initial denaturation at $95{ }^{\circ} \mathrm{C}, 40$ cycles of denaturation at $95{ }^{\circ} \mathrm{C}$ for $5 \mathrm{~s}$ and annealing at $60^{\circ} \mathrm{C}$ for $30 \mathrm{~s}$, and lastly, $1 \mathrm{~min}$ of activation at $95^{\circ} \mathrm{C}$, annealing at $55^{\circ} \mathrm{C}$ for $15 \mathrm{~s}$ and elongation at $95^{\circ} \mathrm{C}$ for $30 \mathrm{~s}$. Each experiment was performed in triplicate. 


\section{In vivo assays}

\section{Biodistribution}

$1 \times 10^{6}$ A549 lung cancer Luc cells, kindly provided by Dr. Anxo Vidal (Santiago de Compostela, Spain), were injected in $0.1 \mathrm{ml}$ PBS into the tail vein of 7-week-old female nu/nu mice (Charles River). The tumor growth was follow-up by in vivo bioluminescence imaging using the Xenogen IVIS (IVISR Lumina II). Mice were anesthetized and injected retro-orbitally with $1.5 \mathrm{mg}$ of $\mathrm{D}$-luciferin $(15 \mathrm{mg} / \mathrm{ml}$ in PBS), images were taken during 5 min with a Xenogen IVIS (IVISR Lumina II) system coupled to Living Image acquisition and analysis software (Xenogen Corporation). For bioluminescence intensity (BLI) plots, photon flux was calculated as previously described [43]. Measurements were performed once a week starting 1 week after tail vein injection and up to 15 weeks. Mice were randomly divided into two groups and treated with miR145-Cy5-loaded F-EMNs $(n=5)$ or miR145-Cy5-loaded exosomes $(n=5)$. Each mouse was injected with a dose of $2.5 \mu \mathrm{g}$ of miR145-Cy5. After $8 \mathrm{~h}$, mice were sacrificed, and the biodistribution of miR145-Cy5 was quantified by ex vivo fluorescence of different organs, lung + tumor, heart, spleen, kidney, and liver, using the using Xenogen IVIS. In each experiment, mice treated with F-EMNs without miR145-Cy5 were used as a control in order to reduce the background tissue.

\section{Confocal analysis in tissue samples}

At the end of the experiment, once the mice were sacrificed and the fluorescence quantified, lung tumors were extracted and frozen in OCT (Tissue-Tek, Sakura). The xenografted tumors were stained with DAPI and Ki67 (Rabbit Anti-Human Ki-67 Monoclonal Antibody (Clone SP6), \#MAD-020310Q, Master Diagnostica) for the identification of metastatic cells. Images were captured in Cy5 emission on an LSM710 Confocal microscope (Zeiss), analyzed and quantified using Fiji software.

\section{Mice handling}

The animal handling and the experimental procedures were approved by the internal ethical research and animal welfare committee (IIB, UAM), and by the Local Authorities (Comunidad de Madrid, PROEX424/15) which complied with the European Union (Directive 2010/63/UE) and Spanish Government guidelines (Real Decreto 53/20133).

\section{Statistics}

Statistical analyses were performed with a GraphPad Prism $^{\circledR}$ software (version 6.0c). All data are expressed as mean \pm standard deviation (SD). Significant differences between two groups were determined by a Student's t-test and multiple comparisons among conditions were done using one-way analysis of variance (ANOVA) followed by Bonferroni post hoc test. * $(\mathrm{p}<0.05),{ }^{* *}(0.05>\mathrm{p}<0.001), * * *(\mathrm{p}<0.0001)$ was considered statistically significant. All experiments were performed at least in triplicate.

\section{Additional file}

Additional file 1. Supplementary data.

Abbreviations

EMNs: exosome-mimetic nanosystems; F-EMNs: functionalized exosomemimetic nanosystem; miR145: microRNA-145; miRscr: microRNA-scramble; integrin a6ß4: integrin alpha-6 beta-4; CLSM: Confocal Laser Scanning Microscopy; FACS: fluorescent activated cell sorting; TEM: transmission electron microscopy; NMR: nuclear magnetic resonance; IP: intraperitoneal; RO: retro-orbital.

\section{Acknowledgements}

We gratefully thank Dr. A Vidal for his technical support for FACS analysis, Dr. SB Bravo (Proteomics Platform, Health Research Institute of Santiago de Compostela) for her technical support for the exosome's protein profiling analysis, Dr. M Martín-Pastor for his technical support in NMR analysis, M Picado for her technical support for confocal imaging and S Alijas for her technical support in the laboratory.

\section{Authors' contributions}

MF conceived and designed the experiments. AVR contributed to the design, conducted the experiments, prepared the figures, and wrote the manuscript. $A M C$ and GMB performed the in vivo experiments. BLB performed cryo-TEM images. All authors discussed the results and commented on the manuscripts. $M F, R L$ supervised the project. MF, GMB, and AMC revised the manuscript. All authors read and approved the final manuscript.

\section{Funding}

This work was in part supported by grants from Instituto de Salud Carlos III (ISCIII) and European Regional Development Fund (FEDER) (CP12/03150, PI15/00828, and CB16/12/00328 from M.F. and PI16/0014, CIBERONC CB16/12/00295 from G.M.B). A.V.R. also acknowledges the financial support from the Spanish Ministry of Education, Culture, and Sport (FPU15/06595).

\section{Availability of data and materials}

The datasets used and/or analysed during the current study are available from the corresponding author on reasonable request.

Ethics approval and consent to participate

Not applicable.

\section{Consent for publication}

Not applicable.

\section{Competing interests}

The authors declare that they have no competing interests.

\section{Author details}

${ }^{1}$ Nano-Oncology Unit, Translational Medical Oncology Group, Health Research Institute of Santiago de Compostela (IDIS), SERGAS, Choupana Street s/n, 15706 Santiago de Compostela, Spain. ${ }^{2}$ University of Santiago de Compostela (USC), Santiago de Compostela, Spain. ${ }^{3}$ Translational Cancer Research Laboratory, Department of Biochemistry, Autonomous University of Madrid, School of Medicine, "Alberto Sols" Biomedical Research Institute CSIC-UAM, IdiPaz, Madrid, Spain. ${ }^{4}$ MD Anderson International Foundation, 28033 Madrid, Spain. ${ }^{5}$ Cancer Network Research (CIBERONC), 28029 Madrid, Spain. 
Received: 2 May 2019 Accepted: 11 July 2019

Published online: 18 July 2019

\section{References}

1. Strebhardt K, Ullrich A. Paul Ehrlich's magic bullet concept: 100 years of progress. Nat Rev Cancer. 2008;8:473-80. https://doi.org/10.1038/nrc23 94.

2. Kibria G, Ramos EK, Wan Y, Gius DR, Liu H. Exosomes as a drug delivery system in cancer therapy: potential and challenges. Mol Pharm. 2018;15:3625-33. https://doi.org/10.1021/acs.molpharmaceut.8b00277.

3. Alvarez-Erviti L, Seow Y, Yin H, Betts C, Lakhal S, Wood MJA. Delivery of siRNA to the mouse brain by systemic injection of targeted exosomes. Nat Biotechnol. 2011;29:341-5. https://doi.org/10.1038/nbt.1807.

4. El-Andaloussi S, Lee Y, Lakhal-Littleton S, Li J, Seow Y, Gardiner C, et al. Exosome-mediated delivery of siRNA in vitro and in vivo. Nat Protoc. 2012;7:2112-26. https://doi.org/10.1038/nprot.2012.131.

5. Tian Y, Li S, Song J, Ji T, Zhu M, Anderson GJ, et al. A doxorubicin delivery platform using engineered natural membrane vesicle exosomes for targeted tumor therapy. Biomaterials. 2014;35:2383-90. https://doi. org/10.1016/j.biomaterials.2013.11.083.

6. Kamerkar S, LeBleu VS, Sugimoto H, Yang S, Ruivo CF, Melo SA, et al. Exosomes facilitate therapeutic targeting of oncogenic KRAS in pancreatic cancer. Nature. 2017;546:498-503. https://doi.org/10.1038/natur e22341.

7. Bai S, Yang T, et al. Exosome delivered anticancer drugs across the blood-brain barrier for brain cancer therapy in Danio Rerio. Pharm Res. 2015:32:2003-14. https://doi.org/10.1007/s11095-014-1593-y.exosome.

8. Rana S, Zöller M. Exosome target cell selection and the importance of exosomal tetraspanins: a hypothesis. Biochem Soc Trans. 2011;39:559-62. https://doi.org/10.1042/BST0390559.

9. Hoshino A, Costa-Silva B, Shen T-L, Rodrigues G, Hashimoto A, Tesic Mark $M$, et al. Tumour exosome integrins determine organotropic metastasis. Nature. 2015:527:329-35. https://doi.org/10.1038/nature15756.

10. Jiang K, Dong C, Yin Z, Li R, Wang Q, Wang L. The critical role of exosomes in tumor biology. J Cell Biochem. 2018;120:1-13. https://doi.org/10.1002/ jcb.27813.

11. Kalluri R. The biology and function of exosomes in cancer. J Clin Invest. 2016;126:1208-15. https://doi.org/10.1172/JCl81135.

12. Vázquez-Ríos AJ, Alonso-Nocelo M, Bouzo BL, Ruiz-Bañobre J, de la Fuente M. Nanotheranostics and their potential in the management of metastatic cancer. In: Condé J, editor. Handbook of nanomaterials for cancer theranostics. Amsterdam: Elsevier; 2018. p. 199-244. https://doi. org/10.1016/b978-0-12-813339-2.00008-6.

13. Llorente A, Skotland T, Sylvänne T, Kauhanen D, Róg T, Orłowski A, et al. Molecular lipidomics of exosomes released by PC-3 prostate cancer cells. Biochim Biophys Acta Mol Cell Biol Lipids. 2013;1831:1302-9. https://doi. org/10.1016/j.bbalip.2013.04.011.

14. Lydic TA, Townsend S, Adda CG, Collins C, Mathivanan S, Reid GE. Rapid and comprehensive 'shotgun' lipidome profiling of colorectal cancer cell derived exosomes. Methods. 2015;87:83-95. https://doi.org/10.1016/j. ymeth.2015.04.014

15. Record M, Carayon K, Poirot M, Silvente-Poirot S. Exosomes as new vesicular lipid transporters involved in cell-cell communication and various pathophysiologies. Biochim Biophys Acta Mol Cell Biol Lipids. 2014;1841:108-20. https://doi.org/10.1016/j.bbalip.2013.10.004.

16. Goh WJ, Zou S, Ong WY, Torta F, Alexandra AF, Schiffelers RM, et al. Bioinspired Cell-derived nanovesicles versus exosomes as drug delivery systems: a cost-effective alternative. Sci Rep. 2017;7:14322. https://doi. org/10.1038/s41598-017-14725-x.

17. Bulbake U, Doppalapudi S, Kommineni N, Khan W. Liposomal formulations in clinical use: an updated review. Pharmaceutics. 2017;9:1-33. https ://doi.org/10.3390/pharmaceutics9020012

18. Alonso-Nocelo M, Abuín C, López-López R, de la Fuente M. Development and characterization of a three-dimensional co-culture model of tumor T cell infiltration. Biofabrication. 2016;8:025002. https://doi. org/10.1088/1758-5090/8/2/025002

19. Zhi D, Bai Y, Yang J, Cui S, Zhao Y, Chen H, et al. A review on cationic lipids with different linkers for gene delivery. Adv Colloid Interface Sci. 2018;253:117-40. https://doi.org/10.1016/j.cis.2017.12.006.
20. Lönn P, Kacsinta AD, Cui X, Hamil AS, Kaulich M, Gogoi K, et al. Enhancing Endosomal Escape for Intracellular Delivery of Macromolecular Biologic Therapeutics. Nat Publ Gr. 2016;6:32301. https://doi.org/10.1038/ srep32301

21. Stremersch S, Vandenbroucke RE, Van Wonterghem E, Hendrix A, De Smedt SC, Raemdonck K. Comparing exosome-like vesicles with liposomes for the functional cellular delivery of small RNAs. J Control Release. 2016;232:51-61. https://doi.org/10.1016/j.jconrel.2016.04.005.

22. Pelt J, Busatto S, Ferrari M, Thompson EA, Mody K, Wolfram J. Chloroquine and nanoparticle drug delivery: a promising combination. Pharmacol Ther. 2018;191:43-9. https://doi.org/10.1016/j.pharmthera 2018.06.007.

23. Mo D, Yang D, Xiao X, Sun R, Huang L, Xu J. MiRNA-145 suppresses lung adenocarcinoma cell invasion and migration by targeting $\mathrm{N}$-cadherin. Biotechnol Lett. 2017;39:701-10. https://doi.org/10.1007/s1052 9-017-2290-9.

24. Zamorano JL, Lancellotti P, Rodriguez Muñoz D, Aboyans V, Asteggiano R, Galderisi M, et al. 2016 ESC Position Paper on cancer treatments and cardiovascular toxicity developed under the auspices of the ESC Committee for Practice Guidelines. Eur Heart J. 2016;37:2768-801. https:// doi.org/10.1093/eurheartj/ehw211.

25. Cirstoiu-Hapca A, Buchegger F, Lange N, Bossy L, Gurny R, Delie F. Benefit of anti-HER2-coated paclitaxel-loaded immuno-nanoparticles in the treatment of disseminated ovarian cancer: therapeutic efficacy and biodistribution in mice. J Control Release. 2010;144:324-31. https ://doi.org/10.1016/j.jconrel.2010.02.026.

26. Peinado H, Zhang H, Matei IR, Costa-Silva B, Hoshino A, Rodrigues G, et al. Pre-metastatic niches: organ-specific homes for metastases. Nat Rev Cancer. 2017;17:302-17. https://doi.org/10.1038/nrc.2017.6.

27. Li P, Kaslan M, Lee SH, Yao J, Gao Z. Progress in exosome isolation techniques. Theranostics. 2017;7:789-804. https://doi.org/10.7150/ thno.18133.

28. Ingato D, Lee JU, Sim SJ, Kwon YJ. Good things come in small packages: overcoming challenges to harness extracellular vesicles for therapeutic delivery. J Control Release. 2016;241:174-85. https://doi.org/10.1016/j. jconrel.2016.09.016.

29. Armstrong JPK, Stevens MM. Strategic design of extracellular vesicle drug delivery systems. Adv Drug Deliv Rev. 2018;130:12-6. https://doi. org/10.1016/j.addr.2018.06.017

30. Jang SC, Kim OY, Yoon CM, Choi D-S, Roh T-Y, Park J, et al. Bioinspired exosome-mimetic nanovesicles for targeted delivery of chemotherapeutics to malignant tumors. ACS Nano. 2013;7:7698-710. https://doi. org/10.1021/nn402232g.

31. Molinaro R, Corbo C, Martinez JO, Taraballi F, Evangelopoulos M, Minardi S, et al. Biomimetic proteolipid vesicles for targeting inflamed tissues. Nat Mater. 2016;15:1037-46. https://doi.org/10.1037/emo00 00122.Do.

32. Li S, Lin Z, Jiang X, Yu X. Exosomal cargo-loading and synthetic exosomemimics as potential therapeutic tools. Acta Pharmacol Sin. 2018:39:542_ 51. https://doi.org/10.1038/aps.2017.178.

33. Parodi A, Molinaro R, Sushnitha M, Evangelopoulos M, Martinez JO, Arrighetti $\mathrm{N}$, et al. Bio-inspired engineering of cell- and virus-like nanoparticles for drug delivery. Biomaterials. 2017;147:155-68. https://doi. org/10.1016/j.biomaterials.2017.09.020.

34. Sato YT, Umezaki K, Sawada S, Mukai S, Sasaki Y, Harada N, et al. Engineering hybrid exosomes by membrane fusion with liposomes. Nat Publ Gr. 2016;6:21933. https://doi.org/10.1038/srep21933.

35. Johnsen KB, Gudbergsson JM, Duroux M, Moos T, Andresen TL, Simonsen $J B$. On the use of liposome controls in studies investigating the clinical potential of extracellular vesicle-based drug delivery systems - a commentary. J Control Release. 2018;269:10-4. https://doi.org/10.1016/j.jconr el.2017.11.002.

36. Serdons K, Verbruggen A, Bormans G. The presence of ethanol in radiopharmaceutical injections. J Nucl Med. 2008;49:2071. https://doi. org/10.2967/jnumed.108.057026.

37. Skotland T, Sandvig K, Llorente A. Lipids in exosomes: current knowledge and the way forward. Prog Lipid Res. 2017;66:30-41. https://doi. org/10.1016/j.plipres.2017.03.001.

38. Swaminathan J, Ehrhardt C. Liposomal delivery of proteins and peptides. Expert Opin Drug Deliv. 2012;9:1489-503. https://doi.org/10.1517/17425 247.2012.735658. 
39. Lv H, Zhang S, Wang B, Cui S, Yan J. Toxicity of cationic lipids and cationic polymers in gene delivery. J Control Release. 2006;1 14:100-9. https://doi. org/10.1016/j.jconrel.2006.04.014.

40. Ozpolat B, Sood AK, Lopez-Berestein G. Liposomal siRNA nanocarriers for cancer therapy. Adv Drug Deliv Rev. 2014;66:110-6. https://doi. org/10.1016/j.addr.2013.12.008.

41. Dubochet J, Adrian M, Chang JJ, Homo JC, Lepault J, McDowall AW, et al. Cryo-electron microscopy of vitrified specimens. Q Rev Biophys. 1988:21:129-228.

42. Kooijmans SAA, Stremersch S, Braeckmans K, De Smedt SC, Hendrix A, Wood MJA, et al. Electroporation-induced siRNA precipitation obscures the efficiency of siRNA loading into extracellular vesicles. J Control Release. 2013;172:229-38. https://doi.org/10.1016/j.jconrel.2013.08.014.
43. Hergueta-Redondo M, Sarrió D, Molina-Crespo Á, Megias D, Mota A, Rojo-Sebastian A, et al. Gasdermin-B promotes invasion and metastasis in breast cancer cells. PLoS ONE. 2014;9:e90099. https://doi.org/10.1371/ journal.pone.0090099.

\section{Publisher's Note}

Springer Nature remains neutral with regard to jurisdictional claims in published maps and institutional affiliations.
Ready to submit your research? Choose BMC and benefit from:

- fast, convenient online submission

- thorough peer review by experienced researchers in your field

- rapid publication on acceptance

- support for research data, including large and complex data types

- gold Open Access which fosters wider collaboration and increased citations

- maximum visibility for your research: over $100 \mathrm{M}$ website views per year

At BMC, research is always in progress.

Learn more biomedcentral.com/submissions 